\title{
2D Correlation Raman Spectroscopy of Hyperbranched Polymer
}

\author{
Ashok. Z. Samuel ${ }^{\mathrm{a}}$, S. Ramakrishnan ${ }^{\mathrm{a}}$, S. Umapathy ${ }^{\mathrm{a}}$ \\ ${ }^{a}$ Inorganic and Physical Chemistry Department, Indian Institute of Science, Bangalore 560012, India
}

IR absorption and Raman spectral bands are very sensitive to changes in interand intramolecular interactions and conformational changes of polymers [1-4]. The traditional analysis of vibrational spectra may result in loss of valuable information on conformational change during melting or crystallization owing to the complexity of the spectral data [5]. 2D correlation analysis of spectral data set proposed by Noda enables a more detailed analysis of spectral variations. In addition to simplifying complex spectral data and improving the spectral resolution, generalized 2D-IR correlation spectroscopy (an extension of the original 2D-CoS) identifies the specific order of certain events occurring under the effect of an external physical or chemical perturbation [6]. 2D-IR correlation spectroscopy has been used earlier to study the subtle conformational modifications of polymer chains during melting and crystallization processes $[7,8]$. In the present study this methodology is applied to study the crystallization and melting behavior of crystallizable hyperbranched polymers. Hyperbranched polymers (HB) are highly branched macro-molecules with features comparable to that of dendrimers. Peripheral functionalized (PEGylated) hyperbranched polyethers made following transetherification methodology has been shown to reorganize in solution [9]. This feature was further utilized to generate self-assembled nanostructures. In order to examine the nature of molecular reorganization, variable temperature IR and Raman spectroscopic studies have been conducted on hyperbranched polymers with crystallizable (Docosanol) units on the periphery (Figure 1).

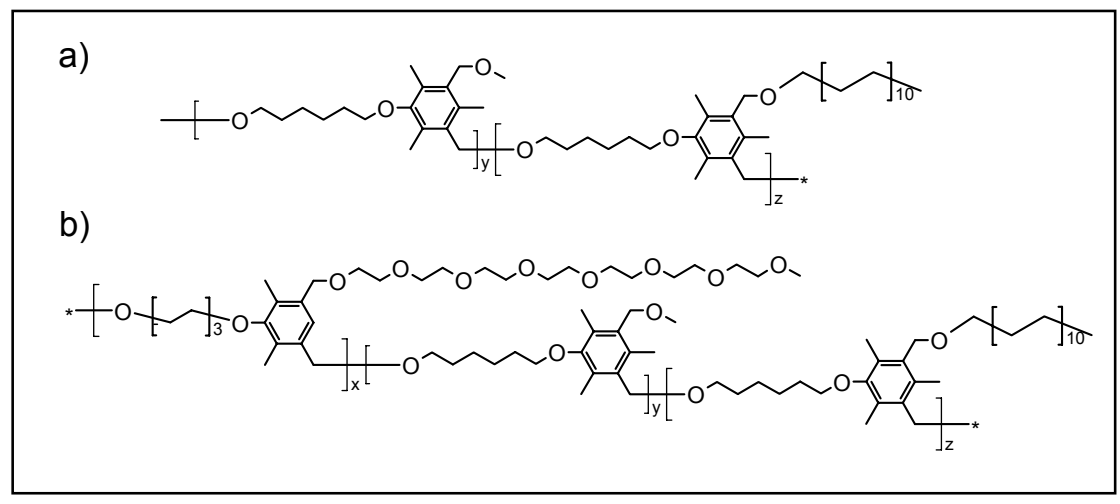

FIGURE 1. Molecular structure of the polymers examined in the present study. a) Hyperbranched polymer functionalized with crystallizable (Docosanol) units at the periphery b) hyperbranched polymer functionalized with polyethylene glycol and Docosanol at the periphery.

The variation in the melting transition of this HB polymer as a function of incorporation of hydrophilic polyethylene glycol (PEG) units are examined using differential scanning 
calorimetric (DSC) measurements. The conformational reorganization during melting and recrystallization of $\mathrm{HB}$ polymer revealed in the $2 \mathrm{D}$ correlation spectra will be discussed and compared with pure Docosanol cystallization and melting events. The variation in the 2D correlation spectrum as a function of incorporation of PEG on to the crystallizable HB polymer will also be examined.

\section{ACKNOWLEDGMENTS}

We would like to thank the IPC department and Indian Institute of Science for the facilities and CSIR for the financial support (scholarship).

\section{REFERENCES}

1. Zhu, X. Y., Yan, D. Y. \& Fang, Y. P. J. Phys. Chem. B 105, 12461 (2001).

2. Jiang, Y., Gu, Q., Li, L., Shen, D. Y., Jin, X. G. \& Chan, C. M. Polymer 44, 3509 (2003).

3. Yan, C., Li, H. H., Zhang, J. M., Ozaki, Y., Shen, D. Y., Yan, D. D., Shi, A. C. \& Yan, S. K. Macromolecules 39, 8041 (2006).

4. Zhu, X. Y., Yan, D. Y., Yao, H. X. \& Zhu, P. F. Macromol. Rapid Commun. 21, 354 (2000).

5. Kai Zheng, Ruigang Liu and Yong Huang, Polymer Journal 42, 81 (2010)

6. Noda, I., Dowrey, A. E., Marcott, C., Story, G. M. \& Ozaki, Y. Appl. Spectrosc. 54, 236A (2000).

7. Peng, Y. \& Wu, P. Y. Polymer 45, 5295 (2004).

8. Zhang, J. M., Tsuji, H., Noda, I. \& Ozaki, Y. J. Phys. Chem. B 108, 11514 (2004).

9. Animesh Saha, S. Ramakrishnan, Macromolecules 41, 5658 (2008) 